\title{
Factors Influencing Technological Capability Building in the Nigerian Solar Energy Industry
}

\author{
Adeyemi Adepoju \\ Federal University of Technology, Akure, \\ Department of Project Management Technology, Akure. Nigeria
}

\begin{abstract}
Technological capability study has continued to gain ground as recognisable progress is being made in the resource-based view (RBV) theory. As a matter of fact, it has become more relevant in the context of a developing nation, such as Nigeria, as a means to create economic development. Using the Panda and Ramanathan methodology for measuring technological capability (TC) in the electric sector, this paper adopted the indicators from the paper to investigate the effects of factors affecting TC on the Nigerian solar energy firms' TCs. The results of seemingly unrelated regressions showed that both internal and external factors significantly affect technological capability in the solar energy industry in different directions, but similar directions to organizational capability of the solar energy firms' in Nigeria.
\end{abstract}

\section{BACKGROUND}

The introduction of renewable energy technologies (RETs) to address the problem of incessant supply and poverty of electricity in both urban and rural areas has been an international development strategy in the developing countries (Tigabu et al., 2015). More so that most RETs are usually diffused product of innovations introduced to the developing nations. Nigeria, a developing nation, is blessed with arrays of renewable energy resources. Among the resources in vast deposit in the country is the solar energy from the sun. But the share of solar energy to the national energy mix is too small compared to the projected estimate envisioned for Nigeria so that its economy can be reckoned with among the twenty largest economies by the year 2020. Based on ECN (2012), the electricity supply projected under the optimistic economic growth rate of $13 \%$ required that by year 2010 and 2015 solar electricity supply would have reached $490 \mathrm{MW}$ and 2,543 MW respectively. Also, that by year 2020, 2025 and 2030 solar electricity supply will be 6,417 MW, 15,970 MW and 39,738 MW respectively. But Sambo (2009) had envisaged earlier that hindrances such as the one relating to technical and financial are great barriers to solar energy technologies proliferation. However, Panda and Ramanathan (1996) opined that the most important task for utilities dealing with electricity in the world especially the developing countries, such as Nigeria, besides financing, seems to be the development of indigenous technological capability. This was reiterated by Vidican (2012) that development of indigenous technological capability in solar energy technologies is required for developing countries to catch-up or leap-frog the developed counter-part. It was also inferred from Akinbami (2001) and Akarakiri et al. (2010) specifically for Nigeria that building technological capability in solar energy technologies become imperative for the indigenous firms in order to provide sustainable electricity supply in the country.

The existing literature widely recognizes the great importance of technological capabilities for long-term sustainable growth, particularly in less advanced countries (Bell, 2010; Ockwell et al., 2010). At the micro-level, technological capabilities are defined as the knowledge and skills that the firm needs to acquire, use, adapt, improve and create technology (Lall, 1992; Bell and Pavitt, 1995). Developing countries, which traditionally lack indigenous capabilities to generate new technologies, commonly rely on diverse mechanisms of international technology 
transfer (Pietrobelli, 2000). Multinational enterprises (MNEs) have played a central role in this process. Indeed, the evolutionary view of technological change, along with intense debate on the globalization of innovation, has drawn increasing attention to MNEs as creators of technology across national boundaries (Iammarino et al., 2008).

Although, research on innovation processes has shown that the technological capabilities held by firms comprise not only information codified in capital goods or documents (patents, manuals, etc.), but also include the tacit knowledge embodied in individual skills and firm routines (Dosi, 1988). These elements of knowledge are costly to transfer and therefore highly organization-specific (von Hippel, 1994). This means that removing trade barriers and providing developing countries with intellectual property rights (IPR) and resources for technology imports is not sufficient to enable countries to catch up to the technological frontier (Ockwell et al., 2010). Rather, catching up requires building local technological capabilities through the cumulative, costly and time-consuming process of technological learning (Bell and Figueiredo, 2012).

Technological capability building is the outcome of complex interactions among individuals, firms, and other organizations within specific institutional frameworks and geographical locations (Edquist and Johnson, 1997). Thus, alongside the technological strategies and profiles of large corporations, the characteristics of local actors and institutional environments are important for determining the impact of MNEs on local capability building (Cantwell and Iammarino, 2003; Iammarino, 2005). Capabilities are built through interactions both within the firm and with external actors (Malerba, 1992). Among these interactions, a crucial role is played by the cooperation in research and development (R\&D) and innovative activities, where such cooperation can involve other firms in the same sector, suppliers, customers, consultants, and scientific institutions like universities and public research centres. The outcomes of these interactions form the basis for the distinction between technological competence and capabilities introduced by von Tunzelmann and Wang (2003). Whilst competences are understood as enhanced inputs to produce goods and services, capabilities generally involve different forms of learning (Malerba, 1992) and the accumulation of new knowledge, eventually embodied in new products and new processes (Iammarino et al., 2012).

Therefore, in order to expand the participation of local companies in more technology intensive activities in the value chain requires technological capabilities (Amsden, 2001). These are critical for enabling developing countries to leapfrog with respect to energy technologies, and incorporate available technologies into their development processes (Goldemberg, 2008). This was also the case with Panda and Ramanathan (1996) on the electricity industry, that the most important task for utilities besides financing, is the development of technological capability.

This study considered the methodology for assessment of technological capability development as suggested in the work of Panda and Ramanathan (1996). Their work considered technological capabilities into three major categories i.e. strategic, tactical, and supplementary capabilities. In addition, they considered another one which is nontechnological, called steering capability. This is included to emphasize the fact that without it technological capabilities cannot be effectively deployed and upgraded. From the procedural steps for the development of a technological capability enhancement plan, this paper examine how the influencing factors affect the technological capability as stated in the work of Panda and Ramanathan (1996). 


\section{METHODOLOGY}

Sampling Technique. The main instrument is a set of structured questionnaire designed to collect information from the firms. Whereas, selection of the sample population required a multistage sampling technique. First, the Yamane formula for proportions at 95 percent confidence level found in Israel (2012) was adopted in the determination of sample size. A simple random sampling technique was used to select 60 firms obtained from a population size of 73 firms found in the business directory belonging to solar energy firms. Thereafter, the second selection of respondents, the unit of analysis was based on purposive selection of one representative each and probability sampling technique of one out of every four technical staff in the solar energy firms. Out of 200 questionnaire administered, 178 questionnaire were retrieved and only 151 of them were considered suitable for the analysis.

Method of Data Analysis. This involves the use of exploratory factor analysis and the use of a regression type known as seemingly unrelated regression analysis.

Exploratory factor analysis (EFA). An EFA may be used for a variety of purposes such as reducing a large number of items from a questionnaire or survey instrument to a smaller number of components, uncovering latent dimensions underlying a data set, or examining which items have the strongest association with a given factor (DiStefano et al., 2009). Many indicator variables were used to understand a construct in this study. So to find an underlying structure to the entire set of latent variables, factor analysis technique was used. The objective was to find means of condensing the information contained in a number of original variables "indicators" to smaller set of variables with a minimal loss of information. Factor analysis provided insight into interrelationships among variables and underlying structure, which is an excellent starting point for many other multivariate techniques. Factor analyses were sequentially used in this study as follow: identifying latent structure, achieving data reduction, variable selection and obtained factor scores for further analysis. The critical assumptions underlying factor analysis are more conceptual than statistical. The overriding concern in factor analysis is much on character and composition of variables included in the analysis as their statistical qualities. The following empirical measures were used to aid diagnosing the factorability of the correlation matrix. Data matrix must have substantial high correlation (more than 0.3), Bartlett test of sphericity provides statistical test for the overall significance of all correlations within a correlation matrix. Also, measure to quantify the degree of intercorrelations among variables and the appropriateness of factor analysis is the measure of sampling adequacy (MSA). This index ranges from 0 to 1 , but it is always advisable that a MSA above 0.5 be achieved before proceeding with the factor analysis.

The selection of extraction method is based on the objective of analysis and amount of prior knowledge about variance of variables. The decision on the number of factors to be retained is usually based on factors eigenvalues greater than 1.0. The oblique rotational method was used in this study because it is more flexible and factor axes will be orthogonal. It is also more realistic because the theoretically important underlying dimensions are not assumed to be uncorrelated with each other (few constructs in the real world are uncorrelated). To further use EFA information in follow-up studies, there was the need to create scores to represent each individual's placement on the factor(s) identified from the EFA. These factor scores were used to investigate the research questions of the study. There are two main classes of factor score computation methods: refined and non-refined. Non-refined methods are relatively simple, cumulative procedures to provide information about individuals' placement on the factor distribution. The simplicity lends itself to some attractive features, that is, non-refined methods are both easy to compute and easy to interpret. Refined computation methods create factor 
scores using more sophisticated and technical approaches. They are more exact and complex than non-refined methods and provide estimates that are standardized scores. In this study, a refined method known as Bartlett approach was used to obtain factor scores based on its advantage over others.

Bartlett's approach, only the shared (i.e., common) factors have an impact on factor scores. The sum of squared components for the "error" factors (i.e., unique factors) across the set of variables is minimized, and resulting factor scores are highly correlated to their corresponding factor and not with other factors. However, the estimated factor scores between different factors may still correlate. Bartlett factor scores are computed by multiplying the row vector of observed variables, by the inverse of the diagonal matrix of variances of the unique factor scores, and the factor pattern matrix of loadings. Resulting values are then multiplied by the inverse of the matrix product of the matrices of factor loadings and the inverse of the diagonal matrix of variances of the unique factor scores.

One advantage of Bartlett factor scores over the other two refined methods (Regression and Anderson-Rubin) is that this procedure produces unbiased estimates of the true factor scores (Hershberger, 2005). This is because Bartlett scores are produced by using maximum likelihood estimates, a statistical procedure which produces estimates that are the most likely to represent the "true" factor scores.

Seemingly unrelated regression (SUR). Based on the factor scores generated for use in objective three predicated the use of seemingly unrelated regression over other regression methods in this study. Basically, multiple regressions describe the behaviour of a particular study variable based on a set of explanatory variables. When the objective is to explain the whole system, there may be more than one multiple regression equations. For instance, in a set of individual linear multiple regression equations, each equation may explain some economic phenomenon. One approach to handle such a set of equations is to consider the set-up of simultaneous equations model is which one or more of the explanatory variables in one or more equations are itself the dependent (endogenous) variable associated with another equation in the full system.

On the other hand, suppose that none of the variables in the system are simultaneously both explanatory and dependent in nature. There may still be interactions between the individual equations if the random error components associated with at least some of the different equations are correlated with each other. This means that the equations may be linked statistically, even though not structurally, through the joint estimates of the distribution of the error terms and through the non-diagonal covariance matrix. Such behaviour is reflected in the seemingly unrelated regression (SUR) equations. In which the individual equations are in fact related to one another, even though superficially they may not seem to be. The basic philosophy of the SUR model is as follows. The joint estimate of the equations is explained by the structure of the SUR model and the covariance matrix of the associated disturbances. Such joint estimate introduces additional information which is over and above the information available when the individual equations are considered separately. So it is desired to consider all the separate relationships collectively to draw the statistical inferences about the model parameters. The model for SUR adapted from Greene (2012) is presented below.

Model:

The model comprises of $\mathrm{M}$ multiple regression equations which can be compactly expressed as

$$
y_{i}=x_{i} \beta_{i}+\varepsilon_{i}, \quad i=1,2, \ldots, M
$$


Where $y_{i}$ is (Tx1) vector with elements $y_{i}$; $X_{i}$ is $\left(T \times K_{i}\right)$ matrix whose columns represents the $T$ observations on an explanatory variable in the $i^{t h}$ equation; $\beta_{\mathrm{i}}$ is a $\left(\mathrm{k}_{\mathrm{i}} \times 1\right)$ vector with elements $\beta_{\mathrm{ij}}$; and $\varepsilon_{\mathrm{i}}$ is a (T $\mathrm{x} 1$ ) vector of disturbances. For the purpose of this study M equals to two (that is the study only has two multiple regression equations). Therefore, when there are two equations the model can be expressed in matrix form.

$$
\begin{aligned}
\left(\begin{array}{l}
y_{1} \\
y_{2}
\end{array}\right) & =\left(\begin{array}{llll}
X_{1} & 0 & \cdots & 0 \\
0 & X_{2} & \ldots & 0
\end{array}\right)\left(\begin{array}{l}
\beta_{1} \\
\beta_{2}
\end{array}\right)+\left(\begin{array}{l}
\varepsilon_{1} \\
\varepsilon_{2}
\end{array}\right) \\
\text { or } \quad y & =X \beta+\varepsilon
\end{aligned}
$$

It is clear that the two equations may appear to be unrelated in the sense that there is no simultaneity between the variables in the system and each equation has its own explanatory variables to explain the study variable. The equations are related stochastically through the disturbances which are serially correlated across the equations of the model. That is why this system is referred to as SUR model.

The SUR model is a particular case of simultaneous equations model with distinct exogenous variables and in which neither current nor logged endogenous variables appear as explanatory variables in any of the structural equations.

The SUR model differs from the multivariate regression model only in the sense that it takes account of prior information concerning the absence of certain explanatory variables from certain equations of the model. Such exclusions are highly realistic in many economic situations.

\section{RESULTS AND DISCUSSION}

Technological capability development in the electricity firm is affected by a number of factors out of which the following internal (technology used, culture, size, strategy, organization structure and management, learning and intelligence gathering, and interest) and external (economy growth, trade regime, fiscal policies and market conditions) factors were considered based on the approach of Panda and Ramanathan (1996). This section is organized into the following subsections: the exploratory factor analyses (EFA) of few constructs required were presented. This is followed by correlation analysis of the variables involved and lastly, the results of seemingly unrelated regressions were provided.

\section{Results of EFA}

In this paper, both technological capability of the firms and factors affecting technological capability accumulation required the use of EFA. Based on the methodology of EFA the results for the constructs are presented.

Table 1 shows the result of factor analysis conducted for technological capability in the solar energy firm using principal component analysis with Oblique Oblimin rotation. The factors analysis revealed two major factors with Eigenvalues above one that jointly explained 62.72 percent of the variation in the original 43 source variables.

The first construct is named the 'technological capability' (tactical) factor as it consists mostly of the tactical capabilities. The other construct identified was termed the 'organizational 
capability' (strategic) (Dutrenit, 2004) because it is closely related to variables mainly aimed at measuring the strategic capability of the firms (Panda and Ramanathan, 1996). In Table 2, the study also conducted a factor analysis of the list of factors affecting the accumulation of technological capabilities in the solar energy industry. Two latent variables were formed from the principal component analysis conducted with oblimin rotation. The first latent variable is named the 'internal factor' as the manifest variables were factors that affect the firms internally (Panda and Ramanathan, 1996). Similarly, the second latent variable obtained from the factor analysis is termed 'external factor' as the manifest variables belong to those affecting the firm from their external environment.

\section{Seemingly unrelated regression analysis for the models}

This sub-section provides report on the regression models for the factors affecting the technological capabilities in the Nigerian solar energy industry. Two variables from the factor analysis conducted, technological capability and organizational capability served as the dependent variables for the two models: disaggregated for model 1 and disaggregated for model 2. The explanatory variables composed of disaggregated factors affecting technological capabilities that include: technology used, company culture, firm size, strategy, structure and management, learning and intelligence gathering, level of interest, size and growth of economy and growth rate, trade regime, financial and fiscal policies of government, interest rate concessions on solar technologies, and market conditions. For the aggregated model, the result of factor analysis produced two factor scores named the internal factor for the first construct, and the second is termed the external factor affecting technological capabilities.

Regression analysis is normally conducted after the correlation analysis results have been examined to avoid the problem of multicollinearity (highly correlated variables). Table 4.36 presents the results of correlation analyses, which is by standardizing the covariance and end up with a value between -1 (perfectly negative relationship) and +1 (perfectly positive relationship) with a coefficient of zero meaning no linear relationship (Field, 2009). In Table 3, the issue of multicollinearity could have been experienced with just a model, but was avoided by having two models. This was noticed with variables such as: Internal and Strategy $(\mathrm{r}=0.880)$; External and Trade $(\mathrm{r}=0.864)$; External and Concession rate $(\mathrm{r}=0.867)$, which therefore necessitate the development of model 1 and model 2.

Table 4 displays the seemingly unrelated regression results for model 1 on factors affecting the technological capabilities in the firms. The results from the table show that the multiple coefficient of determination $\left(\mathrm{R}^{2}\right)$ is 0.94 for technological capability and 0.82 for the organization capability. These are the measure of the goodness of fits of the regression equations or simply the percentage or proportion of the total variations in the dependent variables explained by the explanatory variables. Also, the goodness of fit of the R-squares obtained are further confirmed by the large values of chi-squares obtained that is 2044.29 and 817.63 as well as their lowest significance level at which the null hypotheses could be rejected (p-values) for both technological capability and the organizational capability respectively. The explanation given in the first model is similar for the second model, which is the aggregated factor affecting technological capability in the Nigerian solar energy industry shown in Table 5. In this model, 92 percent of the total variation in the technological capability from the first equation is explained by the total predictor variables. Similarly, the second equation from the table shows that the explanatory variables could predict the proportion of variance in the dependent variable, organizational capability by almost 94 percent. Subsequently, the paper presents the results of partial regression coefficients of the factors affecting technological capabilities in Tables 4 and 5. 


\section{(a) Technology used}

Form the Table 4, it is reported that keeping other factors affecting technological capability constant as well as the effects of correspondence characteristics in addition to the control variables of the firms, the partial regression coefficients for technology used are 0.015 and 1.602 for technological capability and organizational capability respectively. The result shows a positive relationship between the technological capability and technology used which implies that as the level of sophistication of technology used improves technological (tactical) capability also will progress. But this is conversely for the organizational (strategic) capability and technology used as their movement were in opposite directions. It thus appears from the study that both dependent and independent variables have an inverse relationship which signal movement in opposite direction. It is also noted that while the relationship with technological capability (tactical) is not significant ( $p>.05)$ that of the organizational (strategic) does $(\mathrm{p}<.05)$. The implication of these results is that since the technological capability of a firm is influenced by the technology it uses and that learning helps in building technological capability, it is apparent from the results found in objective two that learning in the firms is still subsistence, traditional and lack quality. This may have influenced the obtained results.

\section{(b) Culture of the company}

Since the culture of a company is supportive of technological capability development through continuing emphasis on the development of new skills and information to get new knowledge, it is not surprising that the result obtained on its relationship with technological capability is in opposite direction to each other (Table 4). Although, the result is not significant, it is an evident of the level of technology or knowledge the firms could obtain from our knowledge institutions in the country. Conversely, the cultures of the firms are positively and significantly related to their organizational capability. This is affirming that as the firms strive to open to new knowledge externally, they seem to get none in terms of cutting-edge technology or they are having low absorptive capacity or found similar knowledge obtainable in their firms' also in the system of innovation.

\section{(c) Organization size}

This was measured in terms of total number of employees in the firms. Mostly used as a control variable with the potential of being a positive factor for some capabilities and negative for some others (Panda and Ramanthan, 1996). In this study, firms believe that size is a positive contributing factor to both the technological and organizational capabilities. Table 4 shows that a unit increases in number of reasonable employees will influence the technological capability to increase by a factor of 1.55 . This is also true for the organizational capability as a unit increases in the size of the employees will shift the capability positively by a factor of 0.97 . It implies that size could positively influence the capabilities in the Nigerian solar energy industry and an indication of a policy option for companies' merger or acquisition. This is with the notion that large firms get more opportunity to handle capital intensive projects and hence increase their capabilities.

\section{(d) Organization strategy}

According to Lall (1992), firms are forced to develop higher technological capabilities when they invest progressively in more advanced technology. In case of Table 4 results for the simultaneous equations have shown negative results for both capabilities. Technological capability is found to be significant at 90 percent level ( $p$-value $=0.078$ ) whereas, organizational capability is significant at 95 percent ( $p$-value $=0.028$ ). Firms have shown that presently organizational strategy is moving in opposite direction with technological 
capabilities, which is an indication that there is low rate of major investment in new units of capital-embodied technology in the industry.

\section{(e) Organization structure and management system}

Khandwalla (2001) has revealed that size, degree of vertical integration, complexity of the decision environment and the nature of the technology used by the organization influence the organization's structure and system. This was noticed in the results obtained for this study as well. Table 4 has shown that the result of structure on technological capability positive but not significant $(p>0.05)$ similar to the one obtained in technology used. But different result was obtained for organizational capability as it is positive and significant at 90 percent level. It is an indication that most of the solar firms are small and contributed little to value additions.

\section{(f) Learning and intelligence gathering}

From Table 4 it is evident that both capabilities are negatively related to learning factor with only organizational capability being significant. It is also evident from the table that the magnitude of partial regressions of coefficient of organizational is higher than that of the technological capability. This is true because the strategic arm of the firms belongs to the organizational capability. The negatives signs may have also been the consequences of poor learning and interaction that was also noticed in the results obtained in the second objective.

\section{(g) Interest level}

The results on situational interests depicted positive relationship with both capabilities. It is evident from table 4 that only organizational capability is significant which clearly shows that technological capability requires bringing down the cost of production which entails more involvement in other value addition stages. Other factors contributing to this result on interest may be the market and economy situation in the country. Knowing well that solar energy technologies are still in their formative stages coupled with high cost of production and installation the reception in developing countries such as in Nigeria is still very low compared to other sources of energy which are being subsidized, this may have contributed to the result obtained in the study.

\section{(h) Size of the economy and growth rate}

The size of the economy and growth denoted as 'economy' as shown in table 4 gives negative values for both the technological capability and organizational capability. From the table it can also be deduced that the predictor made significant contributions to both equations. In simple regression, a significant value of $\mathrm{z}$ statistic would mean that the coefficient of regression is significantly different from zero (Field, 2009). The negative results noticed in both technological and organizational capabilities imply that the alternation of long periods of sustained growth with shorter periods of crisis that is expected to improve capability are not true in developing economy like Nigeria. This situation further proves the existence of low level of technological capabilities in the solar energy firms to withstand economic crises such as the increase in inflation rate. It also shows the over-reliance of the industry on importation of solar energy materials from foreign counterpart.

\section{(i) Inward/Outward looking trade regime}

Trade regime measured as the rate at which firms import to locally sourced materials for solar energy technologies. An increase in the measured trade regime affects technological capability negatively. The results in table 4 are not surprising as the firms showed high importation rather than sourcing for materials locally. This is corroborating earlier results stressing the fact that knowledge available is low in the institutions and firms. The results indicate negative partial regression coefficients for both capabilities. But it is more important results to the 
technological capability than it is to the organizational capability as the result is not a significant contributing factor. This is an indication that research on local materials for building technological capabilities need to be emphasized more in our universities and research institutions.

\section{(j) Financial and fiscal policies of government}

Two variables were used to capture financial and fiscal policies of government which are 'fiscal' (rate of import duty on solar technologies) and 'concession rate' (interest rate concessions for solar equipment, materials or components). These are two contrasting variables on both technological and organizational capabilities. For the rate of import duty, the effect is positive on both capabilities with only organizational capability experiencing significant value. The results just mean that it is reasonable decision in terms of strategy for firms locally to incubate and gathered enough capability to face competitions from the foreign ones. But it will rather take a longer period for it to be effective and significant in technological capability. On the second variable, the concession rate positively and significantly affects the technological capability, but insignificant in organizational capability. This stress the fact that Nigerian firms are major importer of these products and nothing are being done locally, again corroborating the result from the trade regime.

\section{(k) Factor market conditions}

Factor market conditions in terms of suitability of local graduates and others affect capability development. The result is significant and has a negative relationship with technological capability. This invariably shows true reflection of the disparity in the level of technological capability in the firms and competence displayed in the innovation system found in the knowledge institutions as revealed in the results presented in objective one. An insignificant result was noticed with the market variable and the dependent variable organizational capability.

\section{(l) Internal and external factors (aggregation)}

Table 5 shows the aggregate results of the factors affecting technological capabilities in the Nigerian solar energy firms. The table has revealed that both the internal and external factors affect the capabilities significantly at 95 percent level. A critical look at the table shows that almost similar contributions were noticed between internal and external coefficients on technological capability but in opposite directions. The aggregate of internal factors affect positively or move in the same direction with technological capability but contrary direction is the case for external factor aggregate. This support the earlier results presented on the relationship between technological capability and trade as well as concession rate. Furthermore, the results of their correlation analysis in Table 3 revealed strong correlation between external and trade as well as concession rate which also reinforced the results obtained. Both internal and external factors were positively and significantly contributing to the accumulation of organizational capability as shown in Table 5. The results of their positive direction with organizational capability is not surprising as strategy factor is strongly correlated with internal factor aggregate, likewise trade regime and concession rate are to external factor aggregate. Invariably, this implies that the firms have strong believe that if this is used as a policy would greatly improve the technological capabilities in the national innovation system on solar energy, firms inclusive. This will make investors to look inwardly and spur researches in our knowledge institutions to support the growth of the indigenous firms. Although, policies of this nature are available in the country there is need to implement and monitor them to stimulate economy growth. 


\section{CONCLUSION}

The study reports some key findings that require critical reasons to be able to provide explanations which are consistent and intuitive. First, the issues of technology used factor having dual relationship with the dependent variables as well as the significant level issues. Supposing we refer to technology as knowledge available within the industry which keeps changing due to the dynamical nature of the environment or the demand-pull effect. It then means that the knowledge available in the Nigerian solar energy is either stagnant or not fast evolving as the case maybe. Secondly, it could also be inferred from the results that since most developing countries such as Nigeria relies mostly on the technology developed by other countries (developed nations). It may then mean that acquiring technological capabilities inline with the absorptive capacity theory as elaborated by Park and Ghauri (2011) where two dimensions are required: firm's prior knowledge base and intensity of effort to learn, need to be rapidly increased. Both the dimensions become highly essential as learning and intelligence gathering results were related negatively. It is possible that overturning the two dimensions can have positive and meaningful results on the organisation's strategy. Likewise, part of the intensity of effort to learning should include policies of the government that would drive international acquisition of local firms as collaborative support by knowledge transferors plays a pivotal role in improving transferees' knowledge capacity to absorb new capability.

\section{References}

Akarakiri, J. B., Ilori, M. O. and Ojo, O. J. (2010). Enhancing Technological Development Through Clustering of Industry, Journal of Research in National Development. 8(1): 1-4.

Akinbami, J. F. K. (2001). Renewable Energy Resources and Technologies in Nigeria: Present Situation, Future Prospects and Policy Framework. Mitigation and Adaptation Strategies for Global Change, 6(2):155-82.

Amsden, A. H. (2001). The Rise of "The Rest": Challenges to the West from Late-Industrializing Economies. Oxford University Press.

Bell, M. (2010). Innovation Capabilities and Directions of Development. STEPS Centre Working Paper 33, Brighton, UK.

Bell, M. and Figueiredo, P. N. (2012). Innovation Capability Building and Learning Mechanisms in Latecomer Firms: Recent Empirical Contributions and Implications for Research. Canadian Journal of Development Studies, 33(1): 14-40.

Bell, M. and Pavitt, K. (1995). The Development of Technological Capabilities, in HAQUEI. U. (Ed.) Trade, Technology and International Competitiveness, pp. 69-101. The World Bank, Washington, DC.

Cantwell, J. A. and Iammarino, S. (2003). Multinational Corporations and European Regional Systems of Innovation. Routledge, London and New York.

DiStefano, C., Zhu, M. and Mîndrilă, D. (2009). Understanding and Using Factor Scores: Considerations for the Applied Researcher. Practical Assessment, Research and Evaluation, 14(20): 1-11.

Dosi, G. (1988). The Nature of the Innovative process. In: Technical Change and Economic Theory, Dosi, G., Freeman, C., Nelson, R., Silverberg, G., Soete, L. (Eds.), Pinter Publishers, London.

Dutrénit, G. (2004). Building Technological Capabilities in Latecomer Firms: A Review Essay. Science Technology Society, 9(2): 209-241.

Edquist, C. and Johnson, B. (1997). Institutions and Organizations in Systems of Innovation, in C. Edquist (ed.) Systems of Innovation: Technologies, Institutions and Organizations. London and Washington: Pinter/Cassell Academic.

Energy Commission of Nigeria- ECN (2012). Renewable Energy Master Plan-updated. Abuja, Nigeria. Field, A. (2009). Discovering Statistics Using SPSS. Third Edition, Sage Publication Limited, City Road, London. Goldemberg, J. (2008). Review The Brazilian Biofuels Industry. Biotechnology for Biofuels. 1(6): 1-7.

Greene, W. (2012). Econometric Analysis. Seventh Edition. Pearson Education Inc., Upper Saddle River, New Jersey, United States of America, 663-743. 
Hershberger, S. L. (2005). Factor Scores. In: Encyclopedia of Statistics in Behavioral Science, Everitt B. S. and Howell D. C. (Eds.), New York, John Wiley, 636-644.

Iammarino, S. (2005). An Evolutionary Integrated View of Regional Systems of Innovation: Concepts, Measures and Historical Perspectives. European Planning Studies, 13(4): 495-517.

Iammarino, S., Padilla, R. and Vontunzelmann, N. (2008). Technological Capabilities and Global-Local Interactions: The Electronics Industry in Two Mexican Regions, World Development. 36: 1980-2003.

Iammarino, S., Piva, M., Vivarelli, M. and Von Tunzelmann, N. (2012). Technological Capabilities and Patterns of Innovative Cooperation of Firms in the UK Regions. Regional Studies, 46(10): 1283-1301,

DOI:10.1080/00343404.2012.679259

Israel, G. D. (2012). Determining Sample Size, Program Evaluation and Organizational Development. IFAS, University of Florida, June PEOD-6, United States America.

Khandwalla, P. N. (2001). Creative Restructuring. Vikalpa, 26(1): 3-18.

Lall, S. (1992). Technological Capabilities and Industrialization, World Development 20(2): 165-186.

Malerba, F. (1992). Learning by Firms and Incremental Technical Change. Economic Journal, 102(4): 845-859.

Ockwell, D., Haum, R., Mallett, A. and Watson, J. (2010). Intellectual Property Rights and LCT Transfer: Conflicting Discourses of Diffusion and Development. Glob Environ Chang. 20(4): 729-738.

Panda, H. and Ramanathan, K. (1996). Technological Capability Assessment of a Firm in the Electricity Sector. Technovation. 16(10): 561-588.

Park, B. I. and Ghauri, P. N. (2011). Key Factors Affecting Acquisition of Technological Capabilities from Foreign Acquiring Firms by Small and Medium Sized Local Firms, Journal of World Business, 46: 116-125.

Pietrobelli, C. (2000). Technology Transfer for Developing Countries. In: Technology Transfer Aldershot: Ashgate, Schroeer, D. and Elena M. (Eds.), 209-234.

Sambo, A. S. (2009). Strategic Developments in Renewable Energy in Nigeria. International Association for Energy Economics. Third Quarters. 2: 15.

Tigabu, A. D., Berkhout, F. and van Beukering, P. (2015). Functional Evolution and Accumulation of Technological Innovation Systems: The Case of Renewable Energy in East Africa. Science and Public Policy. 1-18, doi:10.1093/scipol/scu073.

Vidican, G. (2012). Building Domestic Capabilities in Renewable Energy: A Case Study of Egypt, German Development Institute (DIE). Germany.

von Hippel, E. (1994). Sticky Information and the Locus of Problem Solving: Implications for Innovation. Management Science, 40(4): 429-439.

von Tunzelmann, N. and Wang Q. (2003). An Evolutionary View of Dynamic Capabilities, Economie Appliquée 6: $33-64$. 
Table 1: Factor Loadings for Technological Capability Indicators

\begin{tabular}{|c|c|c|}
\hline \multirow[t]{2}{*}{ Indicator } & \multicolumn{2}{|c|}{ Component } \\
\hline & 1 & 2 \\
\hline Capability to plan, monitor and coordinate marketing and selling activities & .904 & \\
\hline Capability to identify customers, bid and negotiate the terms of service & .896 & \\
\hline Capability to perform production planning & .844 & \\
\hline $\begin{array}{l}\text { Capability to conduct research to determine and monitor customer needs, } \\
\text { wants and satisfaction levels and to set service level standards }\end{array}$ & .798 & \\
\hline Capability to provide training on Solar PV & .764 & \\
\hline $\begin{array}{l}\text { Capability for carrying out preventive, corrective, improving and predictive } \\
\text { maintenance }\end{array}$ & .732 & \\
\hline Capability to provide technical advice to customers & .671 & \\
\hline Capability to do routine design and detail engineering of product or process & .587 & \\
\hline Capability to sell Solar PV technology & .437 & \\
\hline $\begin{array}{l}\text { Percentage of employees in the company sharing the vision of the top } \\
\text { management of the company }\end{array}$ & & \\
\hline $\begin{array}{l}\text { Degree of dependence for the procurement of raw materials, supporting } \\
\text { facilities, spare parts and }\end{array}$ & & .766 \\
\hline Capability to perform civil & & .739 \\
\hline Capability to undertake strategic planning & & .663 \\
\hline Degree of computerization in the firm & & 652 \\
\hline $\begin{array}{l}\text { Capability to identify, assess, negotiate and finalize the terms of the finance to } \\
\text { be acquired without }\end{array}$ & & .570 \\
\hline $\begin{array}{l}\text { Capability to carry out improvements of existing solar PV/CSP } \\
\text { product(s)/process(es) }\end{array}$ & & .463 \\
\hline $\begin{array}{l}\text { Capability to plan, monitor and control construction, erection and } \\
\text { commissioning activities }\end{array}$ & & .409 \\
\hline
\end{tabular}

Table 2: Item Loadings for Factor Affecting Technological Capabilities

\begin{tabular}{lcc}
\hline Item & \multicolumn{2}{c}{ Component } \\
\cline { 2 - 3 } & 1 & 2 \\
\hline Organisation size & .774 \\
Technology used & .762 \\
Organisation structure and management & .740 \\
Company's culture & .737 \\
Learning and Intelligence gathering & .727 & \\
Organisation strategy & .641 & \\
Inward/outward looking trade regime & & .862 \\
Interest rate concessions for solar-PV/CSP equipment, & .819 \\
materials or components & & .686 \\
Financial and fiscal policies of government & & .434 \\
Factor market conditions & & \\
\hline KMO=0.892 & & \\
Total Variance Explained=75.545 percent &
\end{tabular}




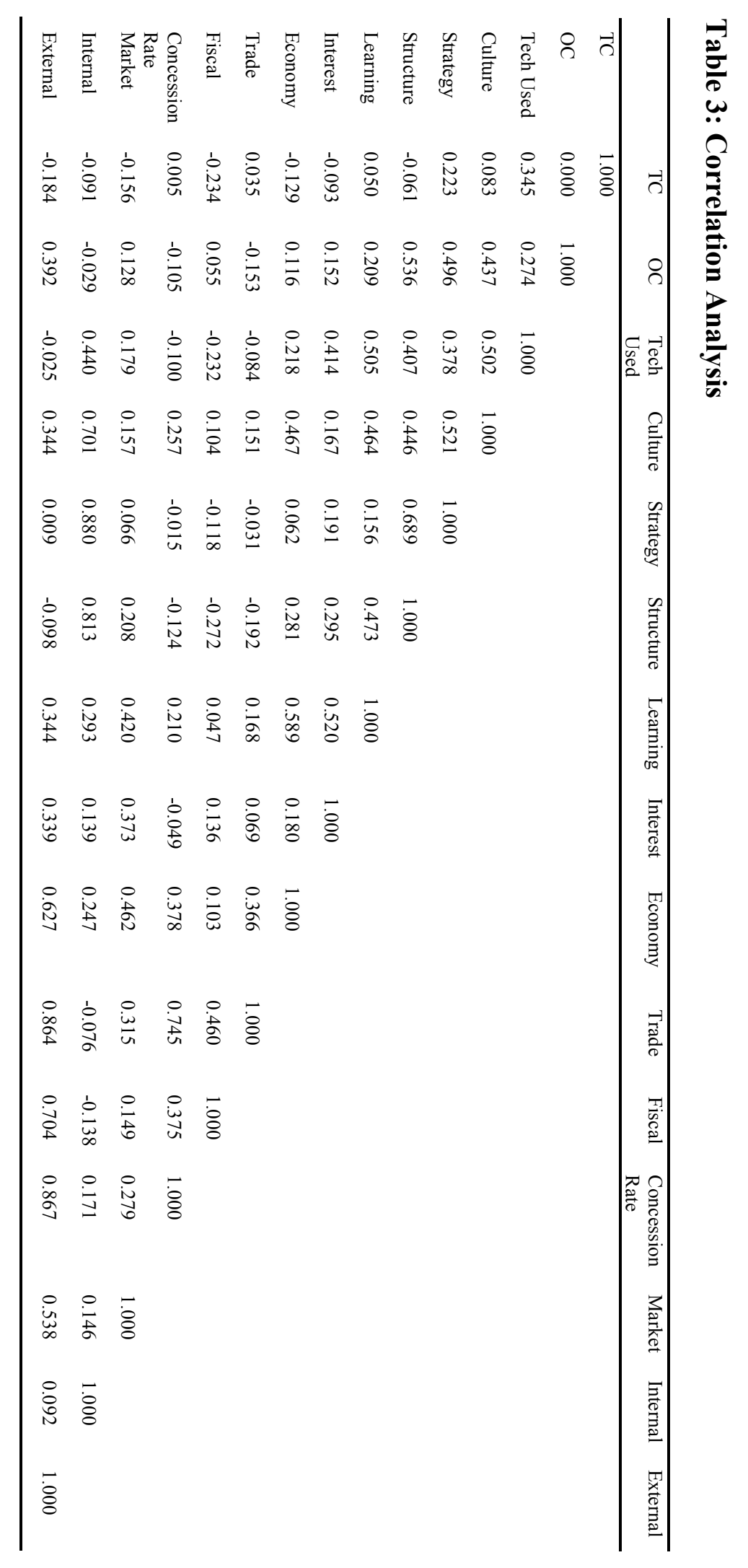


Table 4: Seemingly Unrelated Regression for Model 1 (Disaggregated)

\begin{tabular}{llcccccc}
\hline Equation & Obs & Parms & RMSE & R-Sq & Chi-Sq & P & \\
\hline TC & 151 & 12 & 0.239 & 0.942 & 2044.29 & 0.000 & \\
OC & 151 & 12 & 0.419 & 0.824 & 817.63 & 0.000 & \\
\hline & & & & & & \\
\hline TC & & Coef. & Std. & $\mathbf{z}$ & P>|z| & [95\% Conf. Interval] \\
& & & & & & & \\
& Error & & & & \\
& Tech Used & 0.015 & 0.238 & 0.060 & 0.950 & -0.452 & 0.482 \\
& Culture & -0.403 & 0.334 & -1.210 & 0.228 & -1.057 & 0.252 \\
& TNE & 1.546 & 0.326 & 4.740 & 0.000 & 0.907 & 2.186 \\
& Strategy & -0.445 & 0.253 & -1.760 & 0.078 & -0.941 & 0.050 \\
& Structure & 0.458 & 0.325 & 1.410 & 0.159 & -0.179 & 1.095 \\
& Learning & -0.411 & 0.304 & -1.350 & 0.176 & -1.006 & 0.185 \\
& Interest & 0.281 & 0.334 & 0.840 & 0.400 & -0.373 & 0.934 \\
& Economy & -0.857 & 0.261 & -3.280 & 0.001 & -1.369 & -0.346 \\
& Trade & -0.557 & 0.171 & -3.250 & 0.001 & -0.893 & -0.221 \\
& Fiscal & 0.132 & 0.216 & 0.610 & 0.540 & -0.291 & 0.556 \\
& Concession & 0.647 & 0.282 & 2.300 & 0.022 & 0.095 & 1.200 \\
& Rate & & & & & & \\
Market & -0.468 & 0.171 & -2.730 & 0.006 & -0.803 & -0.132 \\
_cons & -5.093 & 1.788 & -2.850 & 0.004 & -8.598 & -1.588 \\
& & & & & & \\
\hline
\end{tabular}

OC

\begin{tabular}{lcccccc} 
Tech Used & -1.602 & 0.417 & -3.850 & 0.000 & -2.418 & -0.786 \\
Culture & 1.401 & 0.584 & 2.400 & 0.016 & 0.256 & 2.545 \\
TNE & 2.419 & 0.571 & 4.240 & 0.000 & 1.300 & 3.538 \\
Strategy & -0.971 & 0.442 & -2.200 & 0.028 & -1.838 & -0.104 \\
Structure & 1.108 & 0.569 & 1.950 & 0.051 & -0.006 & 2.223 \\
Learning & -1.116 & 0.531 & -2.100 & 0.036 & -2.157 & -0.074 \\
Interest & 2.112 & 0.584 & 3.620 & 0.000 & 0.968 & 3.255 \\
Economy & -1.232 & 0.457 & -2.700 & 0.007 & -2.127 & -0.337 \\
Trade & -0.417 & 0.300 & -1.390 & 0.164 & -1.005 & 0.170 \\
Fiscal & 1.149 & 0.378 & 3.040 & 0.002 & 0.408 & 1.890 \\
Concession & -0.649 & 0.493 & -1.320 & 0.188 & -1.616 & 0.318 \\
Rate & & & & & & \\
Market & 0.258 & 0.299 & 0.860 & 0.390 & -0.329 & 0.845 \\
cons & -3.126 & 3.129 & -1.000 & 0.032 & -9.258 & 3.006 \\
\hline
\end{tabular}


Table 5: Seemingly Unrelated Regression for Model 2 (Aggregated)

\begin{tabular}{|c|c|c|c|c|c|c|c|}
\hline Equation & Obs & Parms & RMSE & $\mathrm{R}-\mathrm{Sq}$ & chi-Sq & \multicolumn{2}{|l|}{$\mathrm{P}$} \\
\hline TC & 151 & 2 & 0.382 & 0.925 & 523.00 & \multicolumn{2}{|l|}{0.000} \\
\hline OC & 151 & 2 & 0.218 & 0.936 & 768.39 & \multicolumn{2}{|l|}{0.000} \\
\hline & & Coef. & Std. Err. & $\mathbf{z}$ & $P>|z|$ & \multicolumn{2}{|c|}{ [95\% Conf. Interval] } \\
\hline \multicolumn{8}{|l|}{ TC } \\
\hline & Internal & 0.740 & 0.148 & 5.010 & 0.000 & 0.451 & 1.030 \\
\hline & External & -0.717 & 0.160 & -4.480 & 0.000 & -1.032 & -0.403 \\
\hline & _cons & 1.579 & 1.375 & 1.150 & 0.251 & -1.116 & 4.274 \\
\hline \multicolumn{8}{|l|}{ OC } \\
\hline & Internal & 0.1971 & 0.081 & 2.430 & 0.015 & 0.038 & 0.356 \\
\hline & External & 0.355 & 0.091 & 3.880 & 0.000 & 0.175 & 0.534 \\
\hline & _cons & -5.236 & 0.785 & -6.670 & 0.000 & -6.774 & -3.698 \\
\hline
\end{tabular}

